\title{
La educación intercultural en Chile analizada desde la teoría de la complejidad
}

\author{
Intercultural education in Chile analyzed from Complexity Theory \\ Gerardo Andrés Fuentes Vilugrón \\ Carlos Roberto Arriagada Hernández
}

\begin{abstract}
RESUMEN
Este ensayo analiza el tema de educación intercultural desde el paradigma de la complejidad, en relación con las prácticas educativas del currículo escolar. El objetivo es analizar las prácticas educativas en relación a los factores que intervienen en el proceso de enseñanza y aprendizaje, desde una mirada intercultural compleja. La metodología consiste en una revisión teórica de la literatura científica y divulgación científica en contextos internacionales, nacionales y regionales. Se discuten algunos elementos que intervienen en la enseñanza-aprendizaje en educación intercultural. Se concluye que la educación intercultural compleja tiene el desafío de hacer efectiva la doble racionalidad educativa en que se interrelacionan todos los saberes, para la construcción de conocimientos y pensamientos propios, tomando en cuenta la diversidad presente en el aula.

Palabras clave: paradigma de la complejidad, interculturalidad, monoculturalidad, multiculturalidad, educación intercultural.

\section{ABSTRACT}

This essay discusses the topic of intercultural education from the paradigm of complexity, in relation to the educational practices of the school curriculum. The objective is to analyze educational practices in relation to the factors involved in the teaching and learning process, from a complex intercultural view. The methodology consists of a theoretical review of the scientific literature and scientific dissemination in international, national and regional contexts. Some elements involved in teaching learning in intercultural education are discussed. It is concluded that complex intercultural education has the challenge of making effective the double educational rationality, in which all knowledge is interrelated, for the construction of knowledge and thoughts of its own, taking into account diversity present in the classroom.

Keywords: paradigm of complexity, interculturality, monoculturality, multiculturalism, intercultural education.
\end{abstract}




\section{INTRODUCCIÓN}

Este ensayo aborda el tema de la educación intercultural, bajo un análisis desde el paradigma de la complejidad aportado por Edgar Morin, en el que el problema a analizar tiene relación con las prácticas educativas y el desarrollo de los contenidos curriculares que proponen la normativa y las políticas públicas aplicadas en la escuela chilena, puesto que, a pesar de las evidencias aportadas por investigadores sociales de la educación, la formación que entrega la escuela chilena es desarrollada desde una "mirada colonialista" o eurocéntrica occidental que promueve la dominación epistemológica de una cultura mayoritaria sobre las minoritarias (Arias, Quintriqueo y Valdebenito, 2018). En este sentido, se adoctrina para la formación homogénea de los estudiantes y se invisibiliza la diversidad social y cultural presente en el aula, que además se caracteriza por la multiplicidad de formas en que se expresa una persona, grupo o sociedad (Herrera, 1991). De esta manera, la formación escolar se desarrolla sin tomar en consideración las diferencias culturales, sociales, religiosas y políticas que tienen influencia sobre el proceso de aprendizaje y enseñanza.

En ese contexto surgen las siguientes interrogantes para comprender el problema: ¿La escuela aborda desde una mirada compleja la educación de sus estudiantes? $¿$ Considera los factores sociales y culturales que intervienen en el desarrollo humano? ¿La teoría de la complejidad es aplicada en los estudios del sistema educativo escolar chileno?

Este artículo sostiene que la educación chilena tradicionalmente se ha centrado en la formación de sus estudiantes sin considerar los factores que intervienen en la construcción de aprendizajes, tales como el contexto, tradiciones, cultura, entre otros (Martínez, 2008). En consecuencia, es posible analizar la educación escolar teniendo como base teórica la teoría de la complejidad planteada por Morin (1990), puesto que se fundamenta en la interrelación y conectividad de factores que intervienen en un proceso o sistema determinado (Álvarez, 2016). En este sentido, al analizar la

Gerardo Andrés Fuentes Vilugrón. Universidad Católica de Temuco, Chile. Es profesor de Educación Física y magister en Didáctica de la Educación Física por la Universidad Autónoma de Chile. Estudiante en el programa de doctorado en Educación en la Universidad Católica de Temuco, Chile. Entre sus publicaciones recientes se encuentran los artículos "El espacio como elemento clave para la regulación emocional en la escuela: análisis en contextos de diversidad social y cultural” (2020) y "Métodos educativos mapuches: retos de la doble racionalidad educativa. Aportes para un enfoque educativo intercultural, de Daniel Quilaqueo Rapimán y Segundo Quintriqueo Millán” (2019). Correo electrónico: gfuentes2019@alu.uct.cl. ID: https://orcid. org/0000-0002-8924-7821.

Carlos Roberto Arriagada Hernández. Profesor asistente de la Facultad de Educación de la Universidad Autónoma de Chile, Chile. Es doctor en Educación. Entre sus publicaciones recientes se encuentran el capítulo de libro "Educación básica en la ruralidad bajo la propuesta de las nuevas bases curriculares" y el artículo "Representaciones del profesorado de la educación básica araucana multigrado sobre las nuevas bases curriculares” (2019). Correo electrónico: carlosarriagadah@gmail.com. ID: https://orcid.org/0000-0002-6707-6451. 
educación intercultural desde lo práctico, se puede evidenciar que presenta ciertas limitaciones en su implementación. Por ejemplo, en la utilización de la lengua española como lenguaje dominante en aquellos contextos de diversidad sociocultural, como una práctica occidental de educar y de formar con base en la homogeneización de los estudiantes, y a través de una única racionalización eurocéntrica occidental, que reproduce valores y prácticas ancladas en aspectos colonialistas y que promueve los modelos educativos indigenistas y de aculturación (Herrera, 1991). Asimismo, el currículo escolar no considera los saberes sociales y culturales indígenas en la construcción de los planes y programas de estudio de la educación escolar chilena (Arias, Quilaqueo y Quintriqueo, 2019), lo que lleva a comprender que la educación intercultural en Chile aún se encuentra en un proceso de construcción y desarrollo para intervenir de mejor manera en el proceso de enseñanza y aprendizaje con contenidos educativos pertinentes y contextualizados a la realidad sociocultural estudiantil. Es decir, un currículo pertinente con las características sociales y culturales para todos los niños, niñas y jóvenes que se desenvuelven en el contexto educativo escolar.

El método de este ensayo, en primera instancia consistió en una revisión de la literatura científica, normativa y de divulgación científica, teniendo como base para el análisis las palabras claves de este ensayo y cómo estas se articulan con la teoría de la complejidad y la educación intercultural en contextos internacionales y nacionales. Posteriormente, para la construcción del corpus del artículo se elabora un recorrido teórico de modo que se comprenda el paradigma de la complejidad y su contextualización en la educación escolar chilena, lo que servirá como marco de análisis de la educación intercultural.

El análisis de la información consistirá en distinguir contenidos sobre los temas expuestos por los autores Herrera (1991), Morin (1990, 1999), Olivé (2011) y Walsh (2009) acerca de la teoría de la complejidad, la educación intercultural y el pluralismo epistemológico, para de este modo examinar antecedentes concluyentes de los temas expuestos en relación con las prácticas pedagógicas presentadas en la educación escolar chilena desde una perspectiva crítica y reflexiva. En este contexto, el objetivo de este ensayo es analizar las prácticas educativas en relación a los elementos que intervienen en el proceso de enseñanza y aprendizaje, desde el paradigma de la complejidad y la educación intercultural.

\section{Paradigma de la complejidad y educación}

Las transformaciones a nivel económico, cultural, político, social, militar, ecológico, jurídico, educativo, entre otros, han provocado un desarrollo mundial denominado "globalización", cuyo concepto está asociado a un fenómeno o proceso caracterizado por abarcar un conjunto de transformaciones en áreas como la economía, la sociología, la política, la cultura, entre otras (Chonchol, 1998; Copelli, 2018; Flores, 2012). Las 
causas de dichos cambios son producto del desarrollo, uso y manejo de tecnologías de la información y las comunicaciones (TICs), puesto que han sido de impulso para la conectividad instantánea del mundo (Rayón, 2018), el sector educativo no ha quedado exento de esto, a través de la incorporación de las TICs como fenómeno inminente y de alta incidencia en la educación (De la Hoz-Franco, Martínez-Palmera, Combita-Niño y Hernández-Palma, 2019). En este contexto, la educación adopta un doble papel, el primero como un espacio impactado, en el que se debe adaptar a los cambios según las nuevas necesidades del mundo, y el segundo como un lugar de reflexión y praxis para discutir, evaluar, analizar y decidir sobre los beneficios y desventajas de la globalización (Avendaño y Guacaneme, 2016).

Para tal efecto, con la globalización y sus infinitas redes que la componen y con el surgimiento de nuevas percepciones y teorías acerca del conocimiento en el mundo, Morin (1990) propone la teoría de la complejidad. Esta teoría puede verse como un paradigma, ciencia, epistemología o método, y es definida como "un tejido de constituyentes heterogéneos inseparables asociados. En un segundo lugar, la complejidad es efectivamente el tejido de los eventos, acciones, interacciones, retroacciones, determinaciones, riegos, que constituyen nuestro mundo" (Morin, 1990, p. 32). En este sentido, la complejidad planteada por Morin se caracteriza por su entrelazamiento dialógico y también por la coexistencia en la heterogeneidad de visiones epistemológicas (De Jesús, Andrade, Martínez y Méndez, 2007).

La teoría de la complejidad trasciende a lo defendido por la simplicidad y el positivismo, puesto que en el positivismo el ser humano es dominador absoluto de la naturaleza, la búsqueda del conocimiento es hacia el interior y personal, y se separa al sujeto de estudio de lo que podría intervenir en la investigación o en los procesos de enseñanza y aprendizaje (Gutiérrez, 2014). Lo anterior se contrasta a lo propuesto por la teoría de la complejidad de Morin (1990), ya que esta busca unir, ligar, entrelazar, articular, contextualizar y entender el desorden y la incertidumbre como algo natural de la realidad, de modo que conecta al ser humano y su conciencia con la sociedad y cultura en que se encuentra.

En síntesis, la teoría de la complejidad busca encontrar una interrelación de todas las cosas, desde una lógica que postula un todo relacional; es decir, el observador no está separado del saber, y el conocimiento va más allá de una relación entre un sujeto y un contenido. Más bien, la complejidad es la interacción de diferentes puntos de vista, que se complementan y oponen al mismo tiempo, sobre algún sujeto y un objeto de conocimiento del mundo (Flores, 2008). Al respecto, no existe una única forma de producir conocimientos o construir aprendizajes, o una sola fuente del conocimiento, más bien la complejidad interrelaciona las diferentes fuentes y métodos para la construcción de nuevos conocimientos. Como plantea Olivé (2011, 2014), la construcción de conocimiento no es relativista ni absolutista sino pluralista, de modo 
que no hay solo un sistema o método válido para el conocimiento humano; sin embargo existe un dominio hegemónico del conocimiento, considerándose los saberes eurocéntricos occidentales como los únicos válidos, superiores y universales, que a la vez inferiorizan e invisibilizan otras formas de producir conocimientos (Herrera, 1991; Walsh, 2009).

Desde el marco de la educación escolar, existen aprendizajes autónomos, individuales y sociales (Perrenaud, 2006), en los cuales se ejercen constantes influencias por los distintos agentes y participantes que están involucrados en la comunidad educativa, a lo que se suman el entorno, el contexto y la cultura en la que está situada la escuela (Martínez, 2008), de modo que dichas influencias no pueden ser aisladas y estudiadas por separado, más bien se necesita vincular los comportamientos y relacionar todos los sistemas que forman parte del contexto escolar (Quezada y Canessa, 2008). En este sentido, este artículo sostiene que, desde el paradigma complejo aplicado a la educación escolar, es preciso ver a todos los individuos que componen la escuela como "sujetos sociales, de influencia recíproca, capaces de establecer, mediante actividades y formas de expresión, determinada red de relaciones e interacciones sociales con el entorno del cual forman parte" (Álvarez, 2016, p. 11). Es decir, la escuela debe buscar la formación, la enseñanza y el aprendizaje comprendiendo el conocimiento de manera multidimensional, que promueva la conciencia, integración y cohesión de saberes socioculturales a través de la educación intercultural entendida como un diálogo de saberes sociales y culturales (Bello, 2014; Malik y Ballesteros, 2015; Quilaqueo, 2019), y que además se considere a los estudiantes como sujetos cognoscentes, con emocionalidad y experiencias que están asociadas al entorno donde se aprende y el contexto al que pertenecen (De Jesús et al., 2007).

También, la educación escolar se puede analizar desde un enfoque transdisciplinar, en el cual se vinculan aspectos demandados como desatendidos a cuestiones urgentes y a retos perennes. Es decir, no solo dar énfasis a una educación para el saber, saber ser y saber hacer, sino, desde un referente de complejidad y conciencia más amplio y ambicioso, brindar una educación para ser mejores personas desde y para el desarrollo integral humano (De la Herrán, 2011).

Tradicionalmente el proceso de enseñanza y aprendizaje en Chile se ha resumido en la entrega de información y contenidos teóricos, mientras que los estudiantes con una participación de oyentes y observadores intentan memorizar los contenidos que proponen los planes y programas de estudio (Fuentealba e Imbarack, 2014). Sin embargo, el proceso de aprender y enseñar consiste en un diálogo con otros y con nosotros mismos, lo que contribuye y promueve un cambio personal antes, durante y después de la enseñanza (Durán, 2014). En relación a esto, el docente agrega a la trasmisión de contenidos el factor motivacional, con el objetivo de tener mayor éxito en el proceso de enseñanza (Sandoval, Mayorga, Elgueta, Soto, Viveros y Riquelme, 
2018). Lo anterior provoca que el profesor visualice el interés de los estudiantes para el aprendizaje, que establezca expectativas de éxito y que desarrolle ayuda adicional para la creación de un ambiente cooperativo, a través de la eliminación de la competitividad en el aula escolar (Ospina, 2006). Así lo expuesto se interrelaciona también con el ambiente escolar que enseña por sí mismo, dado que conlleva la consideración de factores externos como temperatura, ventilación, color de las paredes, cielo raso, luz, decoraciones, recursos materiales, limpieza, entre otros, que ejercen influencias en los aspectos físicos, emocionales, metodológicos y motivacionales de los niños, niñas y jóvenes en la escuela (Castro y Morales, 2015).

En ese contexto, y según las estrategias utilizadas por profesores en Chile, se evidencia un sistema simple de paradigma lineal de causa-efecto; es decir, se busca entregar la mayor cantidad de información sin pérdida de esta, traspasando conocimientos teóricos por sobre el desarrollo de habilidades y actitudes (Arriagada y Calzadilla, 2019), de manera que el profesor tiene como objetivo entregar los contenidos (causa) y el estudiante debe memorizarlos (efecto) sin contextualizarlos a las realidades socio-culturales a las que pertenece. Dicho en otras palabras, y dado que el sistema educativo es lineal, se intenta predecir el comportamiento y rendimiento de los estudiantes en una clase. No obstante, la educación escolar, desde la complejidad, se aleja de este paradigma de tipo lineal, puesto que al interrelacionar las variables provocaría comportamientos colectivos diferentes a lo planificado, en este caso, por el profesor (Quezada y Canessa, 2008).

En relación a lo expuesto por Quezada y Canessa (2008), existen los siguientes elementos y agentes que intervienen en el proceso de aprendizaje y enseñanza en el contexto educativo escolar: motivación, infraestructura, contexto o ambiente de aprendizaje, estudiantes, profesor, sociedad y cultura de pertenencia, política, entre otros. Sin embargo, no se puede reducir la educación a procesos educativos sistematizados, puesto que creer que la educación está sometida solo a los procesos y dinámicas de escolarización es creer en un paradigma simplista. Distinto es considerar los factores externos e internos a la escuela que emergen desde el día en que nacemos a través de los cuidados maternos, relaciones sociales desde el seno familiar y grupo de amigos, entre otros aspectos que contribuyen al desarrollo integral del ser humano (Delors, 1996).

En la educación chilena la formación escolar tiene como objetivo alcanzar el desarrollo espiritual, ético, moral, afectivo, intelectual, artístico y físico, mediante la transmisión y el cultivo de valores, conocimientos y destrezas (Mineduc, 2015). Al respecto, se hace imperativo que la práctica educativa interrelacione todos los elementos que intervienen e influyen en el proceso de enseñanza y aprendizaje como parte de un sistema educativo complejo, puesto que se espera que dicha relación sea de influencia para la construcción de un camino hacia el aprendizaje integral y sig- 
nificativo; es decir, no visto de manera simplista, sino más bien desde un paradigma complejo, en el cual el capital social y cultural en que están inmersas las instituciones educativas escolares, en conjunto con los conocimientos y saberes aprehendidos en el contexto familiar de los estudiantes, se relacionan para desarrollar objetivos de aprendizaje en común.

En síntesis, y según lo expuesto por Morin (1990), la complejidad es la realidad constituida por interacciones (red de vinculación). Por lo tanto cabe señalar que la educación actual está compuesta por diversidad de realidades socioculturales a considerar en el proceso de enseñanza y aprendizaje, tanto por las políticas educativas, equipos de gestión de las instituciones escolares, como también por docentes y estudiantes. Así, para la comprensión de la diversidad social y cultural presente en las aulas se hace necesario profundizar en las diferencias entre los conceptos de multiculturalidad, educación intercultural y educación monocultural.

\section{Multiculturalidad, EDUCaCión MONOCULTURAL Y EDUCACIÓN INTERCULTURAL}

La multiculturalidad es definida por diversos autores como la existencia de varias culturas presentes en un mismo territorio (Quilaqueo y Torres, 2013; Rodríguez, 2018), es decir, esto presenta la diversidad social y cultural dando énfasis en el respeto y la tolerancia. No obstante, la multiculturalidad no ha estado exenta de conflictos y tensiones entre sociedades mayoritarias y minoritarias, lo que ha llevado al desarrollo de la interculturalidad como un planteamiento epistemológico, político y social. De esta manera, se plantea la interculturalidad como un modelo en el cual se promueve la formación que permite ver la realidad desde lo individual y la colectividad, de modo que se facilite la reconfiguración y descentralización cultural, sin discriminar y sin pasar por procesos de aculturación (Castillo y Guido, 2015; Hernández, 2013). Además la interculturalidad tiene como objetivo combatir el etnocentrismo que ha impulsado el colonialismo occidental, el que "tiende a expresar la creencia de que el grupo étnico propio es el más importante, o que la mayoría de sus rasgos de la cultura propia sean predominantes o superiores a los de otros grupos étnicos" (Crúz, Ortíz, Yantalema y Orozco, 2018, p. 180), para la construcción de nuevos esquemas de pensamiento (Castillo y Guido, 2015). Dicho en otras palabras, la educación intercultural hace referencia y propone un intercambio y diálogo de conocimientos culturales entre los sujetos presentes en el sistema escolar (Walsh, 2009).

En contraste a lo anterior, el concepto de monoculturalidad en la educación escolar es definido como "aquella que busca la homogenización del individuo, mediante la transmisión del conocimiento de corte eurocéntrico occidental, considerado como único, válido y científico" (Arias, Quintriqueo, y Valdebenito, 2018, p. 9). Por 
otra parte, se concuerda con lo expuesto por Herrera (1991), puesto que, desde una mirada sociológica, se enseña que los estudiantes deben ser educados desde la heterogeneidad sociocultural, y se debe considerar no solo la lengua de origen o materna sino más bien el lenguaje desde un enfoque más amplio que abarque el razonamiento de la realidad y la apropiación desigual de la realidad que signifique desarrollar una conciencia histórica-social y una conciencia científica, de modo que contribuya a asumir su realidad diferenciada, pero sin connotación de desigualdad, manteniendo su identidad y apropiándose del conocimiento científico para generar una problematización y análisis de la historia y la sociedad.

Sin embargo, la interculturalidad no puede reducirse a una herramienta metodológica, puesto que, desde el marco de la filosofía, la cultura es una experiencia de vida y no una experiencia doctrinal, dado que se caracteriza por la construcción de un sujeto como ser libre, interesada por la producción social y cultural por parte del mismo sujeto (Herrera, 1991); por lo tanto, la interculturalidad es más que un método. Quilaqueo y Torres (2013), en estudios realizados en contextos educativos mapuche, plantean que la educación intercultural es una ontología que se construye con base en la observación e interpretación que hacen aquellos sujetos que comparten un espacio social, cultural y geográfico. No obstante, para que la educación intercultural sea un verdadero diálogo de saberes es necesario primeramente aprender a escuchar, lo que para la cultura occidental eurocéntrica está por debajo del decir. Es por este motivo que, históricamente, los conocimientos y saberes occidentales han sido impuestos a las sociedades minoritarias sin escuchar las necesidades y sin observar los contextos a los que pertenecen las personas (Lilian, 2018).

La educación intercultural vista desde el marco de la teoría de la complejidad no está basada solamente en el conocimiento teórico, más bien abarca una serie de elementos que influyen en los aspectos afectivo, emocional y actitudinal, dado que las respuestas emocionales positivas a la diversidad y la empatía se hacen fundamentales para desarrollarse en este contexto (Peiró y Merma, 2012). Es decir, la educación intercultural, vista desde una mirada de la teoría compleja, se interrelaciona con diferentes criterios, tales como los que expone Van Dijk (2013), en los que incluye el conocimiento intercultural que consiste en el conocimiento social de los problemas, vida cotidiana, experiencias, objetivos y conocimientos propios de cada sociedad y cultura; la comunicación intercultural se refiere a las diferentes formas de idiomas, religiones, normas, valores, costumbres y otras formas compartidas de representaciones y prácticas sociales; la información intercultural expone la tarea de las élites simbólicas de proporcionar información relevante a toda la ciudadanía sobre la sociedad cada vez más diversa; la representación intercultural que consiste en la exigencia de gestión y cooperación entre grupos, líderes y políticos diversos (sociedad democrática), y la toma de decisiones interculturales cuyo enfoque toma en cuenta no solo los intereses 
de la mayoría, sino además las consecuencias sobre los grupos minoritarios. Lo anterior expone la incompatibilidad entre el racismo epistemológico con las sociedades diversas, democráticas e interculturales. Es decir, "la comunicación, la información y la toma de decisiones de las élites simbólicas deben evitar toda forma de estereotipos, prejuicios, discriminación y racismo" (Van Dijk, 2013, p. 119).

$\mathrm{Al}$ respecto, Stefoni, Stang y Riedemann (2016) señalan que producto de esto existen escuelas que se hacen llamar "interculturales", dado que pretenden un encuentro igualitario entre grupos diversos social y culturalmente, mediante el diálogo de conocimientos y saberes. De modo que el diálogo, definido por la Real Academia Española (RAE) como la "plática entre dos o más personas, que alternativamente manifiestan sus ideas o afectos", es la palabra clave en la práctica efectiva de la educación intercultural. Sin embargo, cuando se hace revisión de las políticas públicas y el currículo escolar que se ha desarrollado en Chile se siguen observando escuelas que reproducen un enfoque de adoctrinamiento sociocultural sustentado en la dominación del saber (Herrera, 1991; Stefoni, Stang y Riedemann, 2016; Walsh, 2009). Además se evidencia una contradicción en el trabajo realizado por las instituciones educativas escolares en relación con la normativa planteada en las políticas públicas, debido a que, aun cuando en los documentos curriculares chilenos se declara la importancia de la educación intercultural, sigue existiendo una reproducción de los modelos occidentales en contextos de diversidad sociocultural. Por lo tanto se hace indispensable realizar propuestas y adecuaciones curriculares que acerquen la educación escolar colonizadora a una educación intercultural, no solo pensada en la diversidad social y cultural sino también en la diversidad de género, religión, política, tradiciones, pensamientos y de todas las áreas del ser humano.

\section{Diversidad CUlTuRAL EN LA ESCUELA MIRADA DESDE LA COMPLEJIDAD}

Actualmente ya existe conciencia de que la escuela es un mundo complejo y lleno de diferencias culturales, económicas, sociales, políticas, entre otras (Escarbajal, Mirete, Maquilón, Izquierdo, López, Orcajada y Sánchez, 2012). En este sentido, la escuela tiene el rol de educar, considerando la diversidad, pero logrando otorgar las mismas oportunidades para el aprendizaje de todos los niños, niñas y jóvenes. Además se busca cumplir la misión establecida por la Organización de las Naciones Unidas para la Educación, la Ciencia y la Cultura (UNESCO, 2006) que consiste en:

...cumplir en la promoción de la cohesión social y la coexistencia pacífica. Mediante programas que alienten el diálogo entre estudiantes de diferentes culturas, creencias y religiones, la educación puede contribuir de modo importante y significativo a propiciar sociedades sostenibles y tolerantes [p. 8]. 
En los años recientes han incrementado los índices de matrículas de estudiantes inmigrantes (Fernández, 2018), lo que ha generado un aumento de la diversidad social y cultural dentro del aula, y tener que relacionar la diversidad se ha convertido en un desafío constante para la escuela (Jiménez y Fardella, 2015). Sin embargo, la mirada educativa escolar es de carácter colonizador y homogeneizador que busca chilenizar a estudiantes que provienen de otros contextos socioculturales (Quilaqueo, Quintriqueo, Torres y Muñoz, 2014), de modo que no contribuye a educar desde la diversidad social y cultural, puesto que no hay un intercambio de saberes socio-culturales. Es decir, se observa lo contrario, se adoctrina, se instruye e imponen ideas, conocimientos y la episteme basada en creencias, principios y saberes eurocéntricos occidentales, con el objetivo de que todos los niños, niñas y jóvenes estudiantes alcancen los mismos conocimientos expuestos por los planes y programas de estudio, lo que no considera la complejidad que conlleva la diversidad social y cultural dentro del aula (Walsh, 2009).

De lo expuesto se observa que la educación chilena, según la literatura normativa del currículo escolar, está sustentada en "principios de calidad y equidad educativa, integración, interculturalidad y diversidad para todos y todas, incluyendo a aquellos jóvenes que deben enfrentar barreras que obstaculizan su proceso educativo" (Cornejo, 2017, p. 79). Por consiguiente, la escuela debe evolucionar desde una educación homogeneizadora y segregadora a una educación que responda a todas las necesidades de los y las estudiantes, intentando incorporar los saberes y conocimientos de los pueblos indígenas y de los grupos inmigrantes, con la finalidad de alcanzar una educación integral, de calidad y con pertinencia territorial, social y cultural (Mineduc, 2018).

Por lo tanto, para conseguir esta evolución hay que considerar desde el paradigma de la complejidad y de la educación intercultural a la extensa red de factores que intervienen en la educación escolar chilena, que está compuesta por la relación de los sistemas que la componen, en este caso: escuela, currículo escolar, familia, ambiente, lenguaje, sociedad, cultura, tradiciones, profesores, entre otros. Estos elementos se consideran como los constituyentes más importantes dentro del sistema educativo intercultural y complejo. Por ello Morin (1999) propone educar e interrelacionar los saberes para el desarrollo integral de los estudiantes, abarcando saberes que no han sido expuestos en el contexto de la educación escolar normativa chilena, tales como: (1) el error y la ilusión; (2) conocimientos pertinentes; (3) enseñar la condición humana; (4) enseñar la identidad terrenal; (5) enfrentar las incertidumbres; (6) enseñar la comprensión, y por último (7) la ética del género humano (Morin, 1999). Lo anterior no pretende exponer una serie de materias que debiesen ser enseñadas en la escuela, sino más bien expone los problemas centrales que han sido invisibilizados por la educación escolar chilena en el siglo XXI y que abarcan más allá del currículo asignaturizado, así también pretenden incorporar aquellos aspectos que debiesen ser enseñados para el desarrollo integral de los estudiantes en el presente y en el futuro. 


\section{ConClusiones}

De acuerdo al análisis de la literatura revisada, se evidencia que la teoría de la complejidad tiene relación directa con la educación; es decir, desde el punto de vista de la racionalización heterogénea de la construcción del conocimiento y pensamiento para comprender la realidad y cómo cada uno de estos sistemas o factores están relacionados entre sí para un objetivo en común. Por consiguiente, la interculturalidad en el marco de la educación y la teoría de la complejidad se transforma en más que una propuesta, sino más bien corresponde a un desafío que tiene el objetivo de interrelacionar, ligar o unir cada uno de los aspectos constituyentes que son parte del desarrollo de los estudiantes en el contexto educativo escolar, tomando en consideración la diversidad social y cultural inmersa en el aula.

Según lo expuesto, la educación escolar chilena aún está lejos de considerar la teoría de la complejidad en sus prácticas pedagógicas y estratégicas presentes en la escuela, aun cuando está expuesto de manera explícita en la literatura normativa chilena. En este sentido, las políticas educativas se mantienen en deuda cuando se trata de transmitir contenidos, conocimientos y saberes de forma contextualizada a sus estudiantes caracterizados por la diversidad social y cultural; es decir, que contemplen todas las dimensiones y aspectos que afecten en el proceso de aprendizaje y enseñanza, y por consiguiente en el desarrollo integral de los niños, niñas y jóvenes pertenecientes a diversas sociedades y culturas del país.

Se observa en los antecedentes teóricos analizados que la educación escolar intercultural está también inmersa en un desafío ético, político y epistemológico, ya que desde un ideal teórico y normativo sí se relacionan los elementos que influyen en el aprendizaje de los estudiantes. No obstante, esta propuesta aún está en proceso de construcción y evolución, se pretende que los agentes participantes del proceso de aprendizaje y enseñanza abarquen los saberes formalizados por la escuela y los saberes trasmitidos desde el contexto educativo familiar que se desarrolla en el hogar y en la comunidad a la que pertenecen los estudiantes. Debido a esto, una de las principales influencias es la contingencia cultural cotidiana, pero para ello debe considerarse a la educación intercultural como un modelo multidimensional ligado a la racionalidad de todos los conocimientos, de manera que contribuya en hacer efectiva la doble racionalidad educativa en los estudiantes, interrelacionando los saberes aprehendidos en la educación familiar en conjunto con los saberes aprehendidos en el contexto educativo escolar. Es decir, la educación escolar intercultural y la educación familiar no deben ser vistas como dimensiones aisladas sino como un diálogo de saberes sociales y culturales, para la creación de conocimientos y pensamientos propios de la comunidad estudiantil. De esta manera la formación de los estudiantes será heterogénea y no desde la mirada monocultural y homogeneizante extraída de la educación basada en conocimientos y saberes eurocéntricos occidentales, puesto que solo busca el adoc- 
trinamiento epistemológico e instrucción para que todos los niños y niñas adopten ciertas características sociales y culturales de una sociedad específica mayoritaria que está descontextualizada de la sociedad a la que los estudiantes pertenecen.

Desde la perspectiva expuesta se sostiene que existe la necesidad de formar profesores con un enfoque educativo intercultural visto desde un paradigma complejo, para que en la práctica pedagógica tengan las competencias para formar a los estudiantes desde la diversidad social y cultural presente en el aula, de modo que se haga uso de estrategias que promuevan el diálogo e intercambio de saberes y que contribuyan a prevenir comportamientos de racismo y discriminación entre los estudiantes pertenecientes a diferentes contextos sociales y culturales.

\section{REFERENCIAS}

Alarcón, A., Castro G., M., Astudillo D., P., y Nahuelcheo S., Y. (2018). La paradoja entre cultura y realidad: el esfuerzo de criar niños y niñas mapuche en comunidades indígenas de Chile. Chungará, 50(4), 651-662. Doi: http://dx.doi.org/10.4067/S071773562018005001601.

Álvarez, M. G. (2016). El conocimiento del conocimiento: la obra de Edgar Morin y la problemática de la educación mexicana. IE Revista de la Investigación educativa de la REDIECH, 7(13), 6-20. Recuperado de: http:// www.scielo.org.mx/scielo.php?script $=$ sci_arttext\&pi $\mathrm{d}=\mathrm{S} 2448-85502016000200006$.

Arias, K., Quilaqueo, D., y Quintriqueo, S. (2019). Educación intercultural bilingue en la Araucanía: principales limitaciones epistemológicas. Educação e Pesquisa, 45, 1-16. Doi: https://doi.org/10.1590/ s1678-4634201945192645.

Arias, K., Quintriqueo, S., y Valdebenito, V. (2018). Monoculturalidad en las prácticas pedagógicas en la formación inicial docente en la Araucanía, Chile. Educação e Pesquisa, 44, 1-19. Doi: https://doi.org/10.1590/ s1678-4634201711164545.

Arriagada, C., y Calzadilla, O. (2019). Representaciones del profesorado de la educación básica araucana multigrado sobre las nuevas bases curriculares. Revista Edusol, 19(68), 1-14. Recuperado de: http://edusol. cug.co.cu/index.php/EduSol/article/view/1098.

Avendaño Castro, W., y Guacaneme Pineda, R. (2016). Educación y globalización: una visión crítica. Civilizar.
Ciencias Sociales y Humanas, 16(30), 191-206. Recuperado de: http://www.scielo.org.co/pdf/ccso/v16n30/ v16n30a14.pdf.

Bello Domínguez, J. (2014). Educación intercultural y diálogo de saberes para la paz. Ra Ximbai, 10(2), 175-193. Recuperado de: https://www.redalyc.org/ pdf/461/46132726007.pdf.

Bruner, J. J. (2010). Lenguaje del hogar, capital cultural y escuela. Pensamiento Educativo, (46-47), 17-44. Recuperado de: http://200.6.99.248/ bru487cl/files/ brunner_j.pdf.

Castillo, E., y Guido, S. P. (2015). La interculturalidad: ¿Principio o fin de la utopía? Revista Colombiana de Educación, (69), 17-44. Recuperado de: http:/ / www.scielo.org.co/scielo.php?pid=S0120$39162015000200002 \&$ script $=$ sci_abstract\&tlng $=$ es.

Castro Pérez, M., y Morales Ramírez, M. (2015). Los ambientes de aula que promueven el aprendizaje, desde la perspectiva de los niños y niñas escolares. Revista Electrónica Educare, 19(3), 1-32. Doi: http:// dx.doi.org/10.15359/ree.19-3.11.

Chonchol, J. (1998). Impacto de la globalizacion en las sociedades latinoamericanas: ¿que hacer frente a ello? Estudos Avançados, 12(34), 163-186. Doi: https://doi. org/10.1590/S0103-40141998000300020.

Copelli Ortíz, G. (2018). La globalización económica del siglo XXI. Entre la mundialización y la desglobalización. Estudios internacionales, 50(191), 57-80. Doi: http://dx.doi.org/10.5354/0719-3769.2019.52048. 
Cornejo, C. (2017). Respuesta educativa en la atención a la diversidad desde la perspectiva de profesionales de apoyo. Revista Colombiana de Educación, (73), 77-96. Recuperado de: http://www.scielo.org.co/pdf/rcde/ n73/0120-3916-rcde-73-00077.

Crúz Pérez, M., Ortíz Erazo, M., Yantalema Morocho, F., y Orozco Barreno, P. (2018). Relativismo cultural, etnocentrismo e interculturalidad en la educación y la sociedad en general. Academo. Revista de Investigación de Ciencias Sociales y Humanidades, 5(2), 179-188. Recuperado de: http://scielo.iics.una.py/pdf/academo/ v5n2/2414-8938-academo-5-02-179.pdf.

De Jesús, M., Andrade, R., Martínez, R., y Méndez, R. (2007). Re-pensando la educación desde la complejidad. Polis, Revista Latinoamericana, (16), 1-15. Recuperado de: https://journals.openedition.org/polis/4581.

De la Herrán, A. (2011). Complejidad y transdisciplinariedad. Revista Educação Skepsis, n. 2 Formação Profissional, 1(2), 294-320. Recuperado de: https://radicaleinclusiva.com/wp-content/uploads/2018/01/completrans. pdf.

De la Hoz, F., Martínez-Palmera, O., Combita-Niño, H., y Hernández-Palma, H. (2019). Las tecnologías de la información y la comunicación y su influencia en la transformación de la educación superior en Colombia para impulso de la economía global. Información Tecnológica, 30(1), 255-262. Doi: http:/ / dx.doi.org/10.4067/ S0718-07642019000100255.

Delors, J. (1996). La educación encierra un tesoro. Santillana. Recuperado de http://www.cca.org.mx/apoyos/ competencias/ed5008_009.pdf.

Durán, D. (2014). Aprenseñar: evidencias e implicaciones educativas de aprender enseñando. Narcea Ediciones.

Escarbajal, A., Mirete, A., Maquilón, J., Izquierdo, T., López, J., Orcajada, N., y Sánchez, M. (2012). La atención a la diversidad: la educación inclusiva. Revista Electrónica Interuniversitaria de Formación del Profesorado, 15(1), 135-144. Recuperado de: https://educrea.cl/ la-atencion-a-la-diversidad-la-educacion-inclusiva/.

Fernández, M. P. (2018). Mapa del estudiantado extranjero en el sistema escolar chileno (2015-2017). Chile: Ministerio de Educación. Recuperado de https:/ /www.mineduc.cl/ wp-content/uploads/sites/19/2018/05/MAPA_ESTUDIANTES_EXTRANJEROS_SISTEMA_ESCOLAR_CHILENO_2015_2017.pdf.
Flores, L. (2008). Posiciones y orientaciones epistemológicas del paradigma de la complejidad. Cinta de Moebio, (33), 195-203. Doi: http://dx.doi.org/10.4067/ S0717-554X2008000300003.

Flores, M. V. (2012). La globalización como fenómeno político, económico y social. Orbis. Revista Cientifica Ciencias Humanas, 12(34), 26-41. Recuperado de: https://www.redalyc.org/pdf/709/70946593002. pdf.

Fuentealba, R., e Imbarack, P. (2014). Compromiso docente, una interpelación al sentido de la profesionalidad en tiempos de cambio. Estudios Pedagógicos, 40, 257-273. Doi: http://dx.doi.org/10.4067/S071807052014000200015.

Gutiérrez, R. A. (2014). El paradigma de la complejidad, sus bondades para la reforma de la universidad de San Carlos de Guatemala. Compendium, (33), 43-56. Recuperado de: https://biblat.unam.mx/hevila/ Compendium/2014/no33/3.pdf.

Hernández Collazo, R. L. (2013). Identidad cultural o aculturación: el dilema de una escuela indígena en un contexto urbano. IE Revista de Investigación Educativa de la REDIECH, 4(6). Recuperado de: https: / /www. redalyc.org/pdf/5216/521652344003.pdf.

Herrera, G. (1991). La transmisión del conocimiento y la heterogeneidad cultural. Revista Mexicana de la Sociología, 53(4), 169-182. Recuperado de: https:// www.jstor.org/stable/3540670.

Jiménez, F., y Fardella, C. (2015). Diversidad y rol de la escuela. Revista Mexicana de Investigación Educativa, 20(65), 419-441. Recuperado de: http://www.scielo. org.mx/pdf/rmie/v20n65/v20n65a5.pdf.

Lilian, G. (2018). Hacia una ontología del escuchar. Fundamento del diálogo intercultural. Culturahombre-sociedad, 28(1), 207-233. Doi: http://dx.doi. org/10.7770/0719-2789.3018.cuhso.03.a02.

Malik Liévano, Beatriz, \& Ballesteros Velázquez, Belén. (2015). La construcción del conocimiento desde el enfoque intercultural. Diálogo Andino, (47), 15-25. https://dx.doi.org/10.4067/S071926812015000200003.

Martínez, B. (2008). El aprendizaje de la cultura y la cultura de aprender. Convergencia, 15(48), 287-307. Recuperado de: http://www.scielo.org.mx/scielo.php?script=sci_ arttext\&pid=S1405-14352008000300011. 
Mineduc [Ministerio de Educación] (2015). Proyecto de Ley que crea el Sistema de Educación Pública. Chile: Mineduc. Recuperado de: https://www.mineduc.cl/wp-content/uploads/sites/19/2015/11/ Presentaci\%C3\%B3n-Comisi $\%$ C3\%B3n-deEducaci\%C3\%B3n.pdf (consulta: 17 may. 2019).

Mineduc [Ministerio de Educación] (2018). Programa de Educación Intercultural Bilingüe 2010-2016. Chile: Mineduc. Recuperado de: http://peib.mineduc.cl/ wp-content/uploads/2018/05/20180226-PEIB2010-2016-Versi\%C3\%B3n-Final.pdf.

Moctezuma, E., Yurén, T., y Saenger, C. (2018). La construcción del discurso sobre la diversidad cultural en las universidades públicas del centro-sur de México: una exploración. CPU-e. Revista de Investigación Educativa, (26), 190-213. Recuperado de: https://cpue. uv.mx/index.php/cpue/article/view/2545.

Morin, E. (1990). Introducción al pensamiento complejo. Gedisa. Recuperado de: https://norberto2016.files. wordpress.com/2016/10/morinedgar_introduccional-pensamiento-complejo_parte1.pdf.

Morin, E. (1999). Los siete saberes necesarios para la educación del futuro. UNESCO. Recuperado de: https:// unesdoc.unesco.org/ark:/48223/pf0000117740_spa.

Olivé, L. (2011). Los retos de las sociedades multiculturales: interculturalismo y pluralismo. Cuadernos Intercambios, (9), 207-227. Recuperado de: https:/ / revistas. ucr.ac.cr/index.php/intercambio/article/view/2222.

Olivé, L. (2014). El pluralismo epistemológico y ontológico de Ulises Moulines. Revista Iberoamericana de Ciencia, Tecnología y Sociedad, 10(28), 115-122. Recuperado de: http://www.revistacts.net/volumen10-numero-28/295-dossier/640-el-pluralismo-epistemologico-y-ontologico-de-ulises-moulines.

Ospina, J. (2006). La motivación, motor del aprendizaje. Revista Ciencias de la Salud, (4), 158-160. Recuperado de: https://www.redalyc.org/pdf/562/56209917. pdf.

Peiro, S., y Merma, G. (2012). La interculturalidad en la educación. Situación y fundamentos de la educación intercultural basada en valores. Barataria, (13), 127-139. Recuperado de: http://rua.ua.es/dspace/ handle/10045/35220.

Perrenaud, P. (2006). Construir competencias desde la escuela. Santiago: Noreste. Recuperado de: http:// profordems.uapuaz.com/wordpress/wp-content/ uploads/2011/08/016_perrenoud_03.pdf.

Quezada, A., y Canessa, E. (2008). La complejidad de los procesos educativos en el aula de clases. Educar em Revista, (32), 103-119. Doi: https:// doi.org/10.1590/ S0104-40602008000200009.

Quilaqueo Rapimán, D. (2019). Intervención educativa intercultural para un diálogo de saberes indígena y escolar. Educar em revista, 35(76), 219-237. Doi: https:/ / doi.org/10.1590/0104-4060.63000.

Quilaqueo, D., y Torres, H. (2013). Multiculturalidad e interculturalidad: desafíos epistemológicos de la escolarización desarrollada en contextos indígenas. Alpha, (37), 285-300. Doi: http://dx.doi.org/10.4067/ S0718-22012013000200020.

Quilaqueo, D., Fernández, C., y Quintriqueo, S. (2017). Tipos discursivos a la base de la educación familiar mapuche. Universum, 32(1), 159-173. Doi: http:// dx.doi.org/10.4067/S0718-23762017000100159.

Quilaqueo, D., Quintriqueo, S., Riquelme, E., y Loncón, E. (2016). Educación mapuche y educación escolar en la Araucanía: ¿doble racionalidad educativa? Cadernos de Pesquisa, 46(162), 1050-1070. Doi: https://doi. org/10.1590/198053143599.

Quilaqueo, D., Quintriqueo, S., Torres, H., y Muñoz, G. (2014). Saberes educativos mapuches: aportes epistémicos para un enfoque de educación intercultural. Chungará, 46(1), 271-284. Doi: http://dx.doi. org/10.4067/S0717-73562014000200008.

Quintriqueo, S., y Torres, H. (2013). Construcción de conocimiento mapuche y su relación con el conocimiento escolar. Estudios Pedagógicos, 39(1), 199-216. Doi: http://dx.doi.org/10.4067/S071807052013000100012.

Rayón, M. C. (2018). La globalización: su impacto en el Estado-nación y en el derecho. Revista Jurídica Derecho, 7(8), 19-37. Recuperado de: http://www.scielo. org.bo/scielo.php?script=sci_arttext\&pid=S241328102018000100003\&lng =es\&nrm $=$ iso\&tlng $=$ es.

Rodríguez, H. (2018). La proyección multicultural desde una perspectiva educativa de los derechos humanos. Revista de la Facultad de Derecho, (44), 74-101. Doi: http://dx.doi.org/10.22187/rfd2018n44a3.

Sandoval, M., Mayorga, C., Elgueta, H., Soto, A., Viveros, J., y Riquelme, S. (2018). Compromiso y motivación 
escolar: una discusión conceptual. Revista Educación, 42(2), 66-79. Recuperado de: https://revistas.ucr. ac.cr/index.php/educacion/article/view/23471.

Stefoni, C., Stang, F., y Riedemann, A. (2016). Educación e interculturalidad en Chile: un marco para el análisis. Estudios Internacionales, 48(185), 153-182. Doi: http:/ / dx.doi.org/10.5354/0719-3769.2016.44534.

UNESCO (2006). Durectrices de la UNESCO sobre la educación intercultural. París: UNESCO: https://unesdoc. unesco.org/ark:/48223/pf0000147878_spa.

Van Dijk, T. (2013). Racismo cotidiano y política intercultural. En R. Zapata-Barrero y G. Pinyol Jiménez (eds.), Manual para el diseño de políticas interculturales, (pp. 114-120). Barcelona: Universitat Pompeu Fabra. Recuperado de: https://rm.coe.int/1680301bc4.
Vergara, J. I., y Gundermann, H. (2016). Chile y el Instituto Indigenista Interamericano, 1940-1993. Una visión de conjunto. Chungará, 48(1), 127-144. Doi: http:// dx.doi.org/10.4067/S0717-73562016005000009.

Viveros, L. (2018). Interculturalidad y educación popular: un análisis desde las narrativas de estudiantes universitarios. Sophia Austral, (21), 5-26. Doi: http://dx.doi. org/10.4067/S0719-56052018000100005.

Walsh, C. (2009). Interculturalidad crítica y educación intercultural. En J. Viaña, L. Tapia y C. Walsh, Construyendo interculturalidad crítica (pp. 75-96). La Paz, Bolivia: Intituto Internacional de Integración del Convenio Andrés Bello. Recuperado de: https:// aulaintercultural.org/2010/12/14/interculturalidadcritica-y-educacion-intercultural/.

Cómo citar este artículo:

Fuentes Vilugrón, G. A., y Arriagada Hernández, C. R. (2020). La educación intercultural en Chile analizada desde la Teoría de la Complejidad. IE Revista de Investigación Educativa de la REDIECH, 11, e1107. doi: https:/ /doi.org/10.33010/ ie_rie_rediech.v11i0.1107. 